\title{
Line photon transport in a non-homogeneous plasma using radiative coupling coefficients
}

\author{
R. Florido ${ }^{1,2, *}$, J.M. Gil ${ }^{1,2}$, R. Rodríguez ${ }^{1,2}$, J.G. Rubiano ${ }^{1,2}$, P. Martel ${ }^{1,2}$ \\ and $\mathrm{E}$. Mínguez ${ }^{2}$ \\ ${ }^{1}$ Departamento de Física de la Universidad de Las Palmas de Gran Canaria, \\ Campus Universitario de Tafira, 35017 Las Palmas de Gran Canaria, Spain \\ 2 Instituto de Fusión Nuclear-DENIM, Universidad Politécnica de Madrid, \\ c/ José Gutiérrez Abascal 2, 28006 Madrid, Spain
}

\begin{abstract}
We present a steady-state collisional-radiative (CR) model for the calculation of level populations in non-homogeneous plasmas with planar geometry. The line photon transport is taken into account following an angle- and frequency-averaged escape probability model. Several models where the same approach has been used can be found in the literature, but the main difference between our model and those ones is that the details of geometry are exactly treated in the definition of coupling coefficients and a local profile is taken into account in each plasma cell.
\end{abstract}

\section{INTRODUCTION}

For the most of astrophysical purposes or when we are interested in the diagnosis of laboratory plasmas, the radiation transport cannot be neglected and the emission and reabsorption of photons should be taken into account. Therefore, we face up a complex problem in which an iterative self-consistent procedure between the radiation transfer equation and a set of atomic rate equations is required. Even so there exist elaborated codes which are able to solve this problem for different plasma geometries [1,2]. However for applications where a full line transfer treatment is not necessary, the formalism of escape factors can be used. Actually, in a previous work [3] we used the escape factor formalism proposed in [4] as an approximate way to account for the effects of self-absorption and then we determined the ionic level populations for an homogenous plasma using ATOM3R code [5]. In this work, we approach the line transport in a planar geometry for a non-homogeneous plasma with a finite number of cells, each of them characterized by a different temperature and electron density. Following the ideas developed in [4] and [6], we define radiative coupling coefficients between cells, in which the details of geometry are exactly treated. Using these coefficients, the set of atomic rate equations becomes independent of radiation field. Furthermore, the atomic rate equations are solved using an iterative method and sparse matrices techniques. Finally, calculations of ionic populations are shown for two different spatial temperature and density profiles.

\section{MODEL DESCRIPTION}

We consider a plasma with planar geometry divided along $Z$ axis in $N_{c}$ cells, each of them characterized by an electron density $n_{e}^{c}$ and temperature $T^{c}$, with $c=1, \ldots, N_{c}$. The steady-state rate equation for the

\footnotetext{
*E-mail address: rflorido@dfis.ulpgc.es
} 
population $N_{i}^{a}$ of an atomic level $i$ with ionic charge $\zeta_{i}$ in the cell $a$ is

$$
\frac{d N_{i}^{a}}{d t}=\sum_{j} N_{j}^{a} W_{j i}^{p, a}-N_{i}^{a} \sum_{j} W_{i j}^{d, a}=0
$$

where the population term $W_{j i}^{p, a}$ and the depopulation term $W_{i j}^{d, a}$ are given by

$$
\begin{aligned}
& W_{j i}^{p, a}= \begin{cases}\delta_{\zeta_{i} \zeta_{j}}\left(\Lambda_{j i}^{a} A_{j i}+n_{e}^{a} D_{j i}^{a}\right)+\delta_{\zeta_{i}+1, \zeta_{j}} n_{e}^{a}\left(R_{j i}^{r, a}+R_{j i}^{d, a}+n_{e}^{a} R_{j i}^{3, a}\right) & j>i \\
\delta_{\zeta_{i} \zeta_{j}} n_{e}^{a} E_{j i}^{a}+\delta_{\zeta_{i}, \zeta_{j}+1} n_{e}^{a} I_{j i}^{a} & j<i\end{cases} \\
& W_{i j}^{d, a}= \begin{cases}\delta_{\zeta_{i} \zeta_{j}} n_{e}^{a} E_{i j}^{a}+\delta_{\zeta_{i}+1, \zeta_{j}-1} n_{e}^{a} I_{i j}^{a} & j>i \\
\delta_{\zeta_{i} \zeta_{j}}\left(\Lambda_{i j}^{a} A_{i j}+n_{e}^{a} D_{i j}^{a}\right)+\delta_{\zeta_{i}, \zeta_{j}+1} n_{e}^{a}\left(R_{i j}^{r, a}+R_{i j}^{d, a}+n_{e}^{a} R_{i j}^{3, a}\right) & j<i\end{cases}
\end{aligned}
$$

In the above equations $\delta_{p q}$ is the $\delta$-Kronecker and we have included the corresponding rate coefficients for seven different atomic processes: $I_{i j}$-electron impact ionization, $R_{j i}^{3}$-three-body recombination, $R_{j i}^{r}{ }^{-}$ radiative recombination, $R_{j i}^{d}$-dielectronic recombination, $E_{i j}$-electron impact excitation, $D_{j i}$-electron impact deexcitation and $A_{j i}$-spontaneous emission. In (2) and (3) the escape factor $\Lambda_{j i}^{a}$ has been introduced. This quantity gives the probability that a photon emitted in the cell $a$ as a consequence of the radiative decay $j i(i<j)$ escapes from the plasma without being absorbed. The escape factor represents a way to take into account the effects of the emission and reabsorption of photons and it is defined through the following expression

$$
N_{j}^{a}\left(A_{j i}+B_{j i} J_{j i}^{b b}\right)-N_{i}^{a} B_{i j} J_{i j}^{b b}=N_{j}^{a} \Lambda_{j i}^{a} A_{j i}
$$

where $J_{i j}^{b b}$ is the frequency-averaged mean intensity of the radiation field for a bound-bound transition.

The escape factor $\Lambda_{j i}^{a}$ can be written in the following way

$$
\Lambda_{j i}^{a}=C_{j i}^{a}-\sum_{c=1, c \neq a}^{N_{c}} \frac{N_{j}^{c}}{N_{j}^{(a)}} C_{j i}^{a c}
$$

In the last expression, the first term gives the probability that a photon emitted in the cell $a$ escapes from it without being absorbed. The summation can be interpreted as the probability that the photon is absorbed in any of the remaining cells. Following the ideas described in [4] and [6], we have found the following expressions for the radiative coupling coefficients for a plane-parallel medium

$$
\begin{aligned}
C_{j i}^{a} & =\int_{\nu} \phi_{j i}^{a}(\nu)\left[\frac{1}{2 \tau_{a}}+\frac{1}{\tau_{a}} F\left(\tau_{a}\right)\right] d \nu \\
C_{j i}^{a c} & =\int_{\nu} \phi_{j i}^{a}(\nu) \frac{1}{2 \tau_{a}}\left[F\left(\tau_{a c}+\tau_{c}+\tau_{a}\right)-F\left(\tau_{a c}+\tau_{c}\right)-F\left(\tau_{a c}+\tau_{a}\right)+F\left(\tau_{a c}\right)\right] d \nu
\end{aligned}
$$

where $F(\tau)=\frac{1}{2}\left[(\tau-1) e^{-\tau}-\tau^{2} E_{1}(\tau)\right], E_{1}(\tau)$ is the first-order exponential integral, $\tau_{a}$ is the optical depth of the cell $a$ and $\tau_{a c}$ is the optical depth between cells $a$ and $c$. In the above equations the details of geometry are exactly solved. In addition, as the frequency integration is approached as the last step in the procedure, a specification of the profile function before the geometry-dependent integrals is no longer needed and the consideration of more sophisticated profile functions (i.e. to include Stark effect) becomes feasible.

We have found the equation (6) under assumption of some approximations. It was assumed that complete frequency redistribution holds, so absorption and emission profiles becomes equal for each line in a given cell. We also considered that there is no overlapping between different lines and continuumradiation effects were not included. 
This procedure represents an approximate way to take into account the line photon transport and it may be employed to obtain reliable solutions to the coupled equations of radiative transfer and level kinetics.

\section{APPLICATION TO NON-HOMOGENEOUS PLASMAS}

To show the usefulness of our model, we have calculated the spatial distribution of level populations and average ionization in a non-homogeneous aluminum plasma with planar geometry (see Figure 1).

We carried out the calculations over two different pairs of density and temperature profiles, which were provided by hydrodynamic simulations performed with the 2D-hydrodynamic code ARWEN [7]. One of the cases presents a strong gradient in temperature and density (Figure 2, left) while the other one shows a smooth variation (Figure 3, left). ATOM3R is able to handle large sets of atomic data by the use of sparse matrices techniques and algorithms [8]. However, in this work the calculations were done using an atomic database which includes only 325 levels for ions from neutral to full stripped aluminum, because in this case we only wanted to analyse the effects of including the radiative transfer model. On the other hand, we used well-known analytical formulas to get the rate coefficients of the different atomic processes. To show the suitability of the proposed model, we have carried out three different types of calculations:

No-radiation, i.e. we consider completely uncoupled cells and we also consider that they are thin, so any absorption effect is not included in the model.

No-coupling, i.e. we consider completely uncoupled cells but we take into account only the probability that one photon emitted in a given cell is absorbed in the same cell.

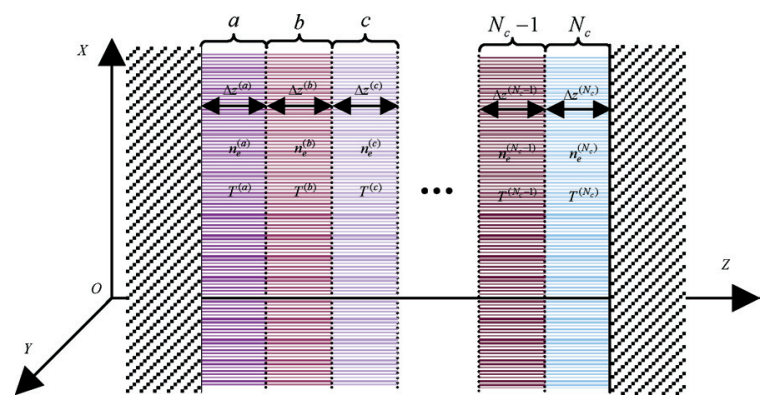

Figure 1. Schematic representation of the plane-parallel medium in the radiative coupling coefficients model.
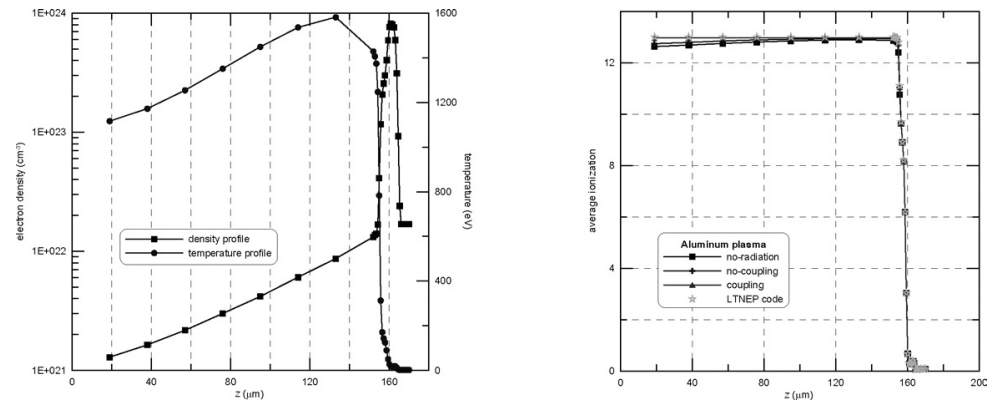

Figure 2. Spatial electron density and temperature profiles with a strong gradient (on the left). The corresponding spatial average ionization distribution for the three types of mentioned calculations (on the right). 

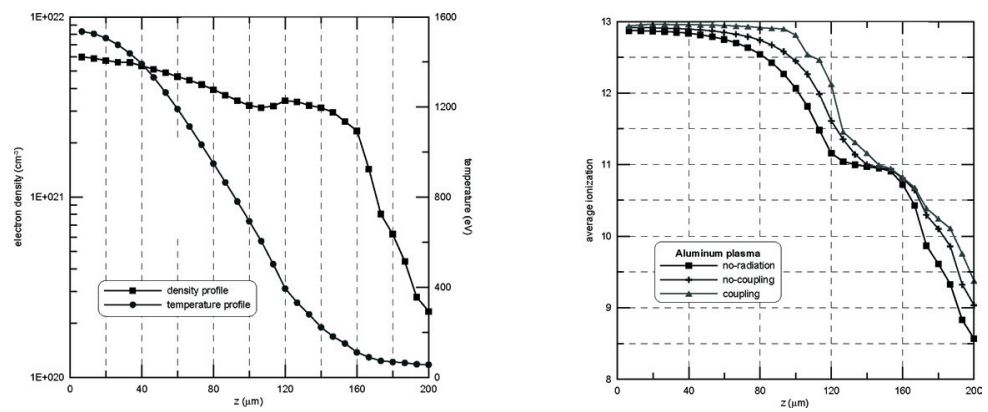

Figure 3. Spatial electron density and temperature profiles for the second proposed case (on the left). The corresponding spatial average ionization distribution for the three types of mentioned calculations (on the right).

Coupling, i.e. the model described in this work. We take into account the probability that one photon emitted in a given cell can be absorbed in any of the remaining cells. For each bound-bound transition we calculate a radiative coupling coefficient for every pair of cells $a b$.

In Figures 2 and 3 (right) we represent the average ionization spatial distribution obtained with the three described types of calculation for the mentioned density and temperature profiles. It can be seen that an increase of the average ionization takes place as more elaborated models are used to take into account the radiation effects. In addition, in the first case we observe an excellent agreement between our model and LTNEP code [3], which solves the time independent 1D equation transfer self-consistently with rate equations. On the other hand, we have also found a significant redistribution of the population of excited states in the different plasma cells when the line photon transport is taken into account. This is an important subject to be considered when the population distribution has been determined thinking of a later calculation of radiative properties such as absorption or emission spectrum.

\section{Acknowledgments}

This work was partially supported by the grant AI-62 from the program INNOVA 2003 of the Fundación Universitaria de Las Palmas and by the project ENE-2004-081084-CO3-0/FTN from the Ministerio de Educación y Ciencia (Spain).

\section{References}

[1] H.A. Scott, J. Quant. Spectrosc. Radiat. Transfer 71 (2001) 689-701.

[2] O. Peyrusse, Phys. Fluids B 4 (1992) 2007-2017.

[3] P. Sauvan, R. Florido, J.M. Gil, R. Rodríguez, J.G. Rubiano, P. Martel, E. Mínguez, R.C. Mancini, F. Ogando, in Proceedings of the 17th International Conference on Spectral Line Shapes, Paris, 2004, edited by E. Dalimier (Frontier Group, Paris, 2004) 230-237.

[4] R.C. Mancini, R.F. Joyce, C.F. Hooper, J. Phys. B: At. Mol. Phys. 20 (1987) 2975-2987.

[5] R. Florido, J.M. Gil, R. Rodríguez, J.G. Rubiano, P. Martel, E. Mínguez, in Proceedings of the 28th European Conference on Laser Interaction with Matter, Rome, 2004, 388-413.

[6] J.P. Apruzese, J. Davis, J. D. Duston, K.G. Whitney. J. Quant. Spectrosc. Radiat. Transfer 23 (1980) 479-487.

[7] F. Ogando, P. Velarde, J. Quant. Spectros. Radiat. Transfer 71 (2001) 541-550.

[8] R. Florido, J.M. Gil, R. Rodríguez, J.G. Rubiano, P. Martel, E. Mínguez, in Proceedings of the 32nd EPS Plasma Physics Conference, Tarragona, 2005, edited by C. Hidalgo, B.Ph. van Milligen (European Physical Society, 2005) P-5.124. 